\title{
ELECTROMYOGRAPHY OF THE ERECTOR SPINAE IN LOW BACK PAIN
}

BY J. S. R. Golding

From the Orthopaedic Department, The Middlesex Hospital

The ligaments of the lower back and of the ankle are injured more often than those of any other region. Whereas the mechanism of injury and the treatment of ankle injuries are clearly defined, however, the mechanism of injury of the lower back and its treatment are still highly controversial subjects.

The ligaments and bones of the lower back are protected by muscle action. Any force which tends to injure the ligaments sets up a powerful stretch reflex in the erector spinae which is usually strong enough to protect them from injury. Recently, with the development of electromyographic techniques, a considerable body of evidence has accumulated concerning the behaviour of the erector spinae under different conditions. This knowledge helps our understanding of the way the back may be injured and the way the upright posture is maintained; it is also important as a guide to the management of low back injuries and to the prevention of recurring attacks of pain.

The work to be described has been performed using the action potentials produced in the erector spinae and recording them by means of surface electrodes. There is an exact correlation between records made in this way and those recorded with

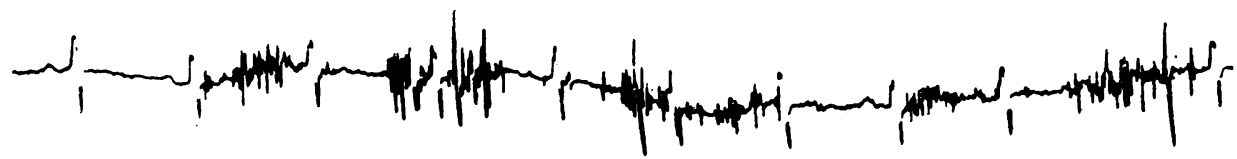

L. ERECTOR SPINAE I. 2-3

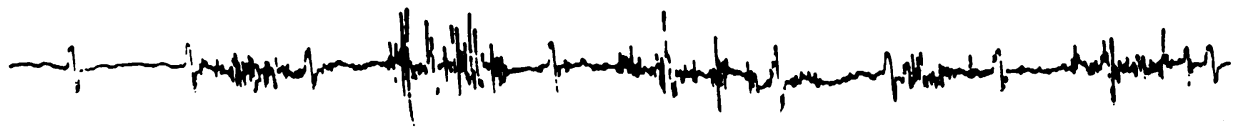

L. ERECTOR SPINAE L. 3-4
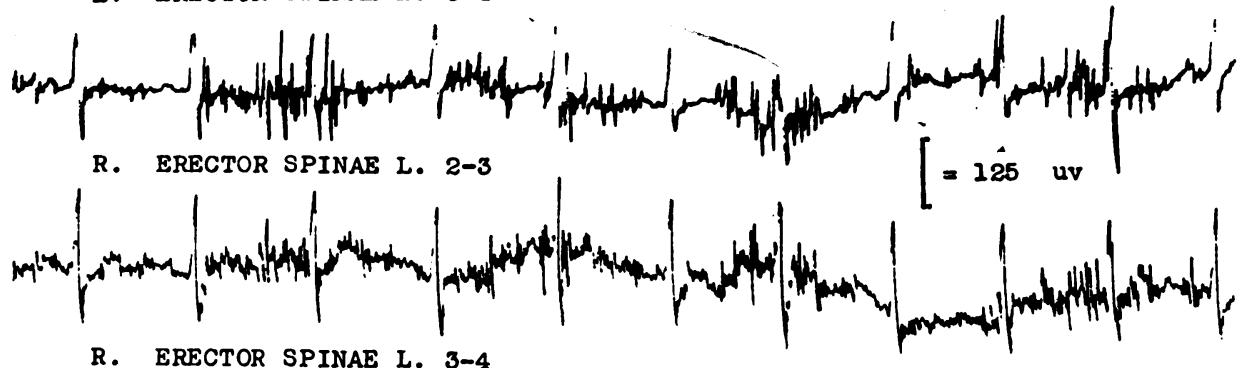

R. ERECTOR SPINAE L. 3-4

1 SEC

FIG. 1.-The erector spinae in standing, showing a low level of intermittent activity. The electrocardiogram can be clearly seen. 


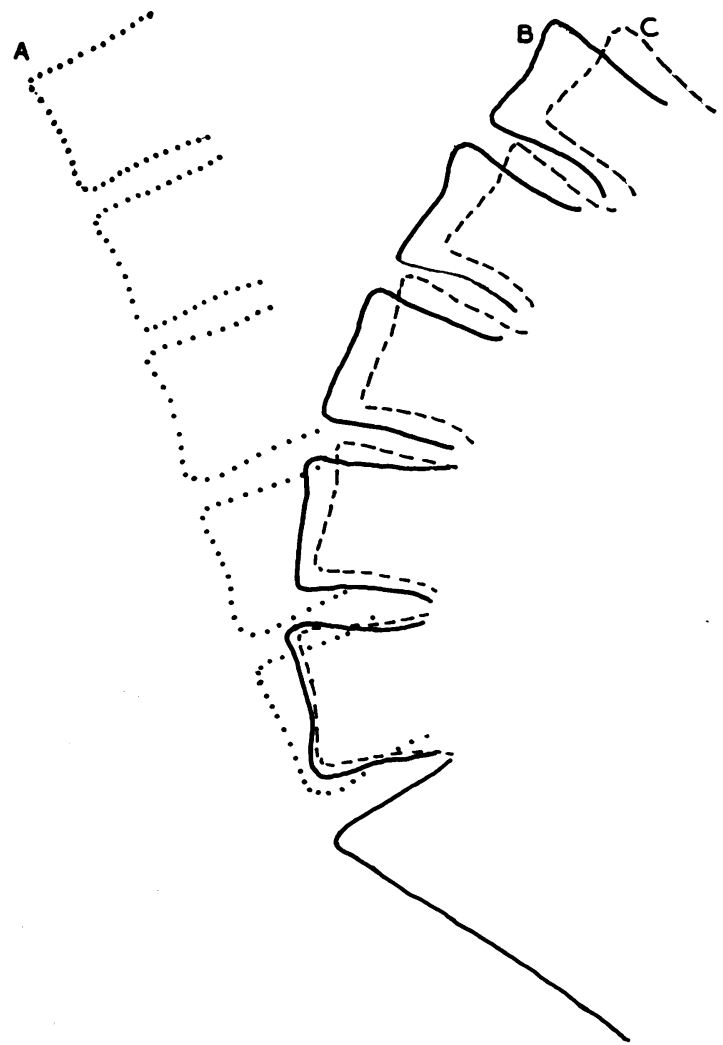

needle electrodes, because no muscle fibres overlie the erector spinae in the lower lumbar region. The recordings were made from the erector spinae muscles on both sides at two levels between the second and fifth lumbar spines.

\section{Standing at Rest}

When a normal subject stands upright, very little activity is found in the erector spinae. The record shown in Fig. I was taken from a normal man standing relaxed and shows a general level
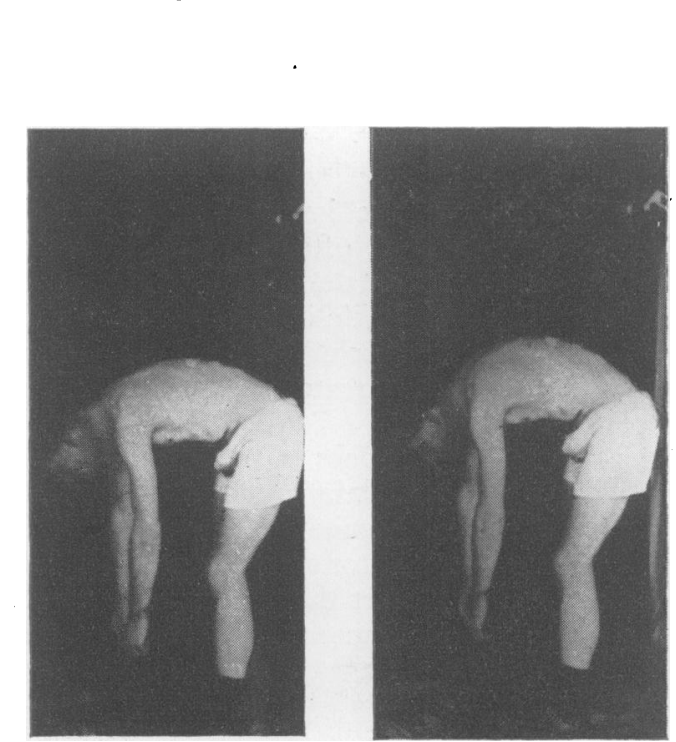

Fig. 2.- Tracings of lateral radiographs of the lumbar spine and sacrum taken with the patient standing : A. Fully flexed.

B. Erect.

C. Fully extended.
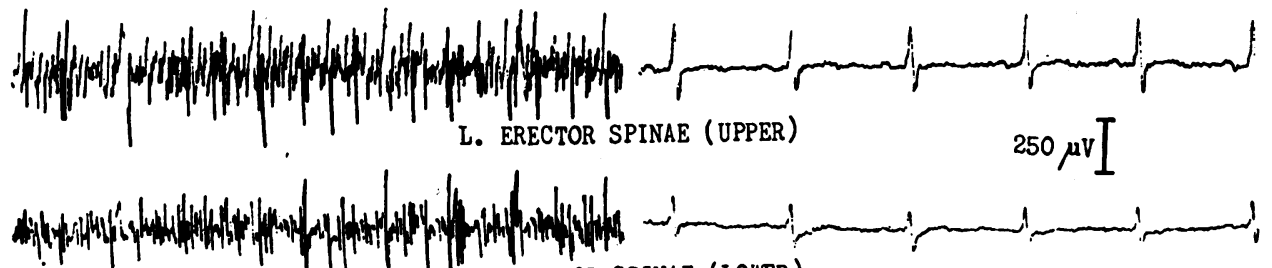

L. ERECTOR SPINAE (LOWER)
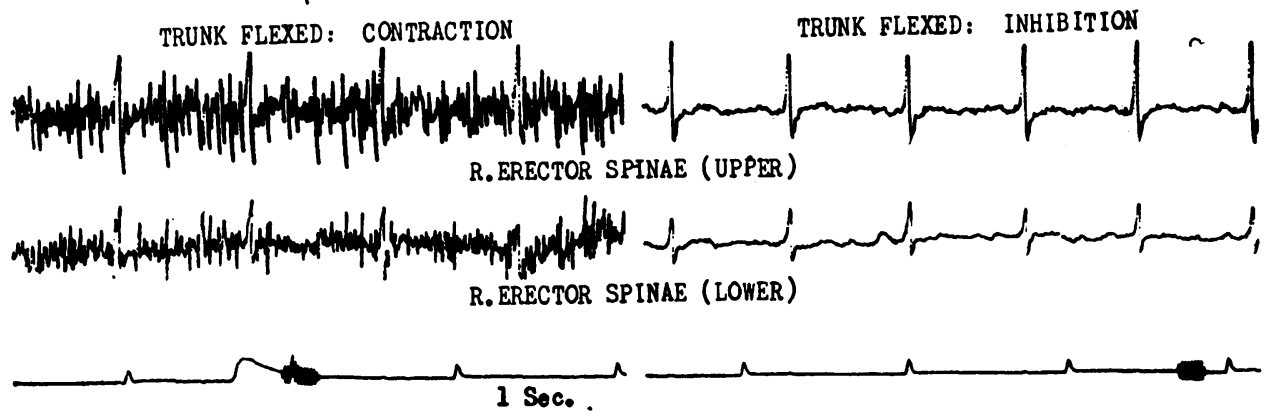

Inhibition of Erector Spinae in Trunk Flexion

Fig. 3.-Electromyogram to show the activity of the erector spinae in bending forward. In the left record, a deliberate effort is made to extend the lumbar spine, without extending the hip joints. In the right record, the erectors are allowed to relax once more. Notice the very slight difference in the contour of the back. 
of slight activity. These tracings show small intermittent bursts of firing which represent the reaction of the erector spinae to a slight forward swaying of the trunk. This amount of activity in the erector spinae is too slight by itself to account for the maintenance of the erect posture, and it is obvious that the main weight of the body must be taken by the vertebrae, intervertebral discs and ligaments. In standing, the lower back is extended (Fig. 2); the posterior intervertebral joints are near one limit of their range of movement, just like the hip and knee joints. The centre of gravity of the body lies vertically above and just behind the centre of the hip joint, in front of the point of contact of femoral and tibial condyles and just in front of the ankle (Meyer, 1873). This position allows the weight in the lumbar region to be taken mainly by the anterior common ligament and intervertebral discs (Akerblom, 1948). Normal erect posture may thus be defined as a position adopted by the body where the weight is taken mainly by the vertebrae and intervertebral ligaments and discs, where muscle activity is slight and where the intervertebral joints are near to, but not quite at, the fully extended position.

\section{Bending Forwards}

As the normal subject begins to bend forwards, the erector spinae contracts powerfully to control the movement. Then, as full flexion is reached, the erector spinae suddenly relaxes (Fig. 3). This relaxation is usual in normal subjects although not invariable, and some records show only partial inhibition of the muscle (Floyd and Silver, I95I). When this inhibition has occurred and the subject is instructed to resume the upright position slowly, some movement at the hip joints takes place and then suddenly the erector spinae begins to contract again.

This mechanism of powerful contraction followed by relaxation of the erector spinae during flexion is of clinical importance. If a load falls on the shoulders whilst the erector spinae is contracting, the muscle protects the ligaments from injury. However, when the trunk is fully flexed and the erector spinae fully or partially relaxed, the ligaments are unprotected and particularly subject to injury.

Failure of Flexion Relaxation
An investigation of 120 patients complaining

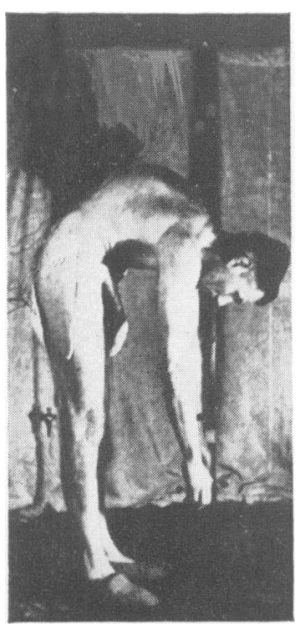

Ankylosing Spondylytis: Relaxation of Erector Spinae

Fig. 4A.-Electromyogram of erector spinae from a patient with ankylosing spondylitis, showing relaxation of the erector spinae with 'trunk flexion.'
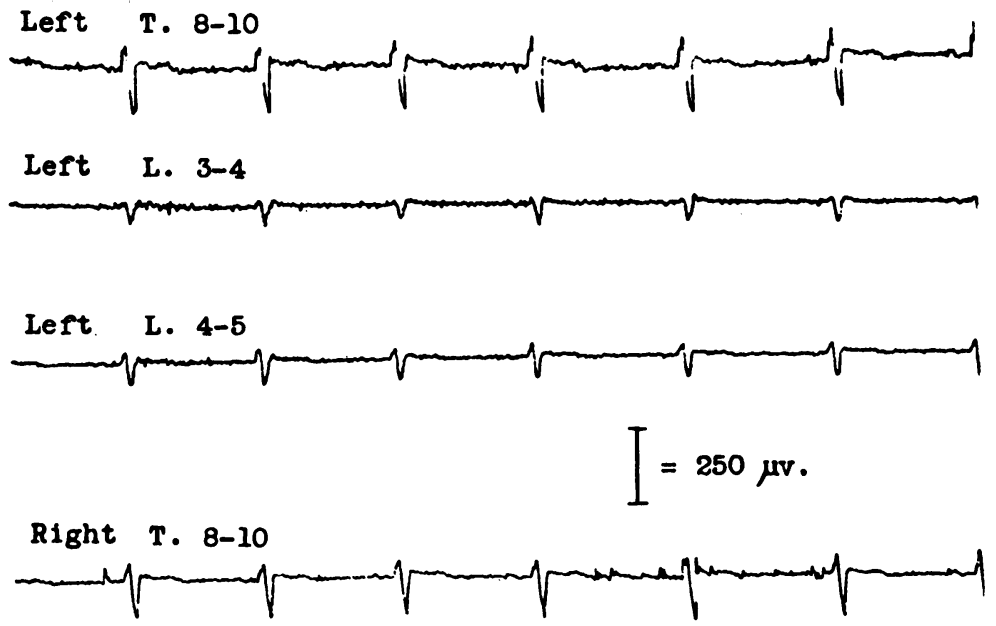

Right L. 3-4

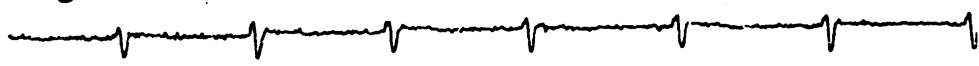

Right I. 4-5

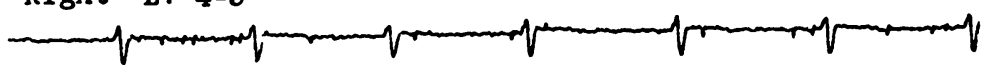

1 sec.

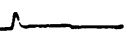


of low back pain was made to find out if any abnormal action could be detected in the activity of the erector spinae. It became apparent that gross structural disease, provided there was no pain, was compatible with normal relaxation in flexion (Figs. $4 a$ and b). However, where the diagnosis of injury or disease of the intervertebral ligaments or discs was made clinically, flexion relaxation did not occur. There were 29 patients who stated that their pain started following a flexion injury of the lower back; of these, 26 showed complete failure of relaxation of the erector spinae on full flexion. In all, there were 34 patients in whom complete failure of relaxation on full flexion was found; of these, 25 had either a prolapsed intervertebral disc or a strain of the posterior interspinous ligaments (Newman, 1952), 5 had organic vertebral disease such as tuberculosis or active ankylosing spondylitis, and the remaining 4 were thought to be suffering from lumbasacral strain.

From these figures, it is apparent that there is a close correlation between a flexion injury, failure to relax the erector spinae on attempting full flexion, and painful ligamentous injuries of the lower back. Following on a flexion injury, the erector spinae contracts to protect the injured area from strain in the same way as the evertors of the foot prevent any attempt at inversion after a strain of the lateral ligament of the ankle joint. This protective contraction allows repair to proceed with the torn ends of the ligaments in close

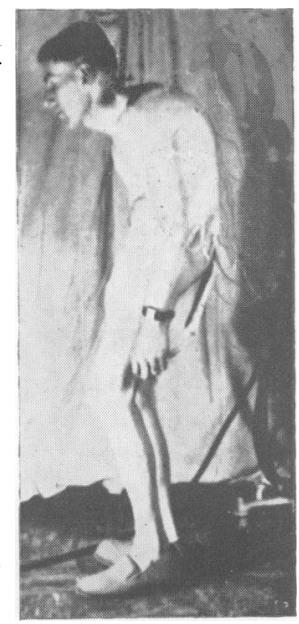

Ankylosing Spondylitis: Upright Stance

Fig. 4B.-Electromyogram of erector spinae from a patient with ankylosing spondylitis, showing activity in the erector spinae in the upright stance.

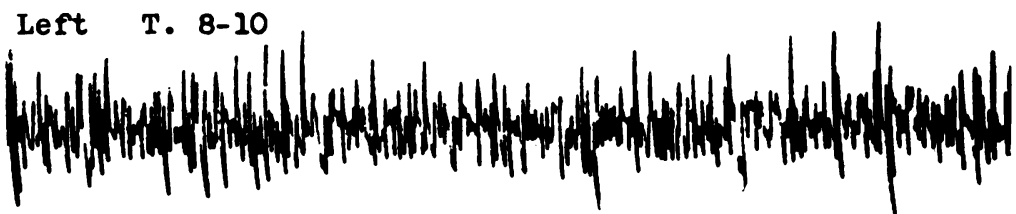

Left I. 3-4
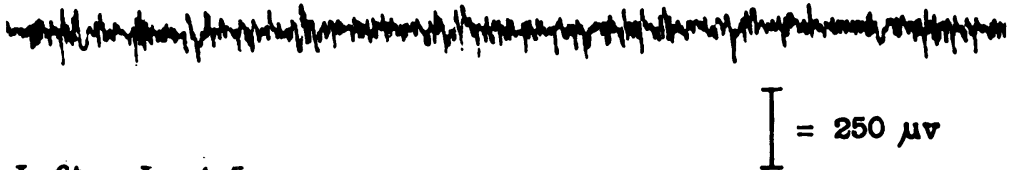

Left L. 4-5

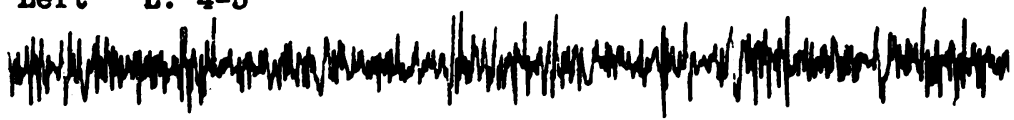

Right T. 8-10

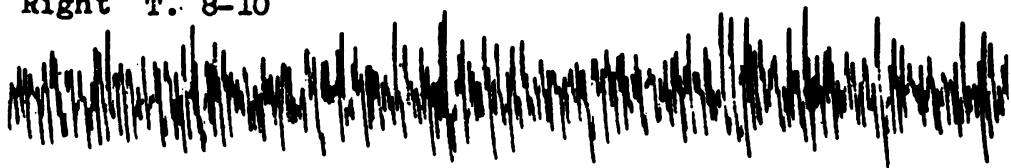

R1ght I. 3-4

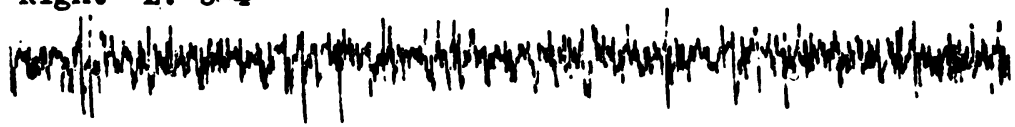

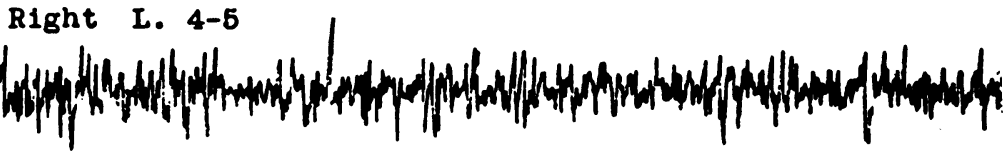

1 sec. 


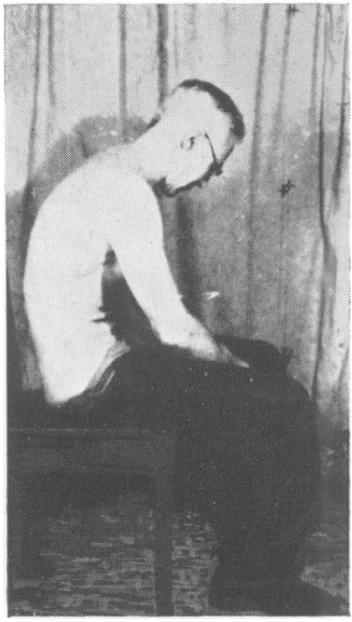

Erector Spinae Relaxation in Sitting

FIG. 5.-Electromyogram of erector spinae in a patient with a history of old injury to the intervertebral disc, showing relaxation in flexion while sitting.
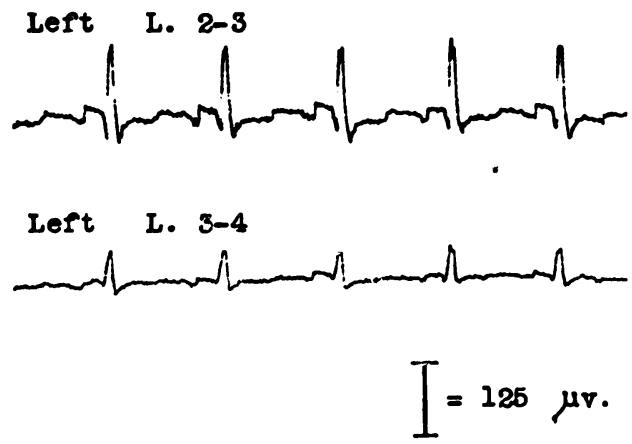

R1ght I. 2-3
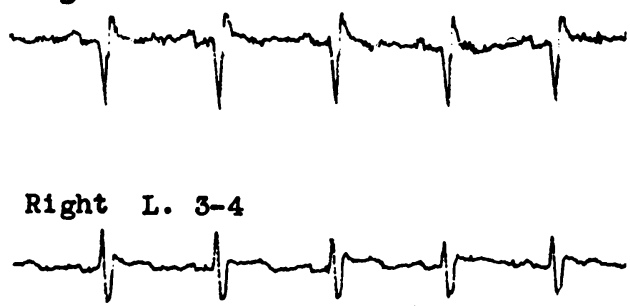

1 sec. apposition. Any movement which does not disturb the injured area is painless ; persistent pain may be regarded as good evidence that repair is not occurring satisfactorily.

As the lesion heals and becomes painless, this protective contraction or "spasm " of the erector spinae gradually passes off and an intermediate condition is reached (Figs. 5 and 6). This patient, suffering from prolapse of an intervertebral disc and responding to conservative treatment, showed failure of flexion relaxation when standing; however, when sitting, with the load on the intervertebral disc considerably less, he was able to relax the lumbar spine fully.

\section{Influence on Treatment}

Authorities are agreed that the correct treatment for injuries of the ligaments of the lower back is, in the first instance, rest to the part (O'Connell, 1950). The problem is how best to secure rest. As shown above, the erector spinae contracts to prevent strain on the injured ligaments and to allow healing to take place. Accordingly, the position which avoids the need for this protective contraction of the erector spinae and the pain which causes it is the ideal position for treatment.

Usually a prolapsed disc heals best when the patient lies in bed with the lumbar spine in slight extension. In this position the posterior longitudinal ligament is relaxed, because the posterior margins of the bodies of the vertebrae become approximated. The axis of vertebral rotation during the movement of flexion and extension lies well behind the centre of the intervertebral discs, so that in extension the volume of the disc space is increased and it becomes easier for any material displaced backwards to re-enter the disc space. Once the displaced annulus is back in the disc space, the posterior lips of the vertebral bodies tend to prevent any re-displacement.

The conservative plan of treatment which is recommended for patients suffering from acute ligamentous injuries of the lower back is based on this conception of the aetiology of the condition. Most patients find very rapid relief of pain from lying on a firm mattress with one pillow under the head and another in the small of the back. If pain is relieved by this treatment within a week, extensor back exercises are commenced and $\mathrm{q}$ week later, if there is still little or no pain, a plaster jacket is applied. This jacket is designed to hold the lumbar spine in extension ; it should extend well up to the manubrium above and to the pubis below, but may be quite narrow posteriorly over 


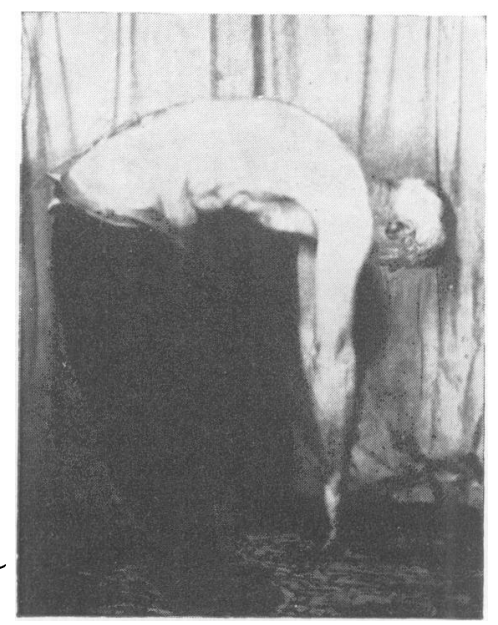

Maximum Flexion: Failure of Relaxation in Standing

Fig. 6.-Electromyogram of erector spinae in a patient with a history of old injury to the intervertebral disc, showing failure of relaxation in standing.
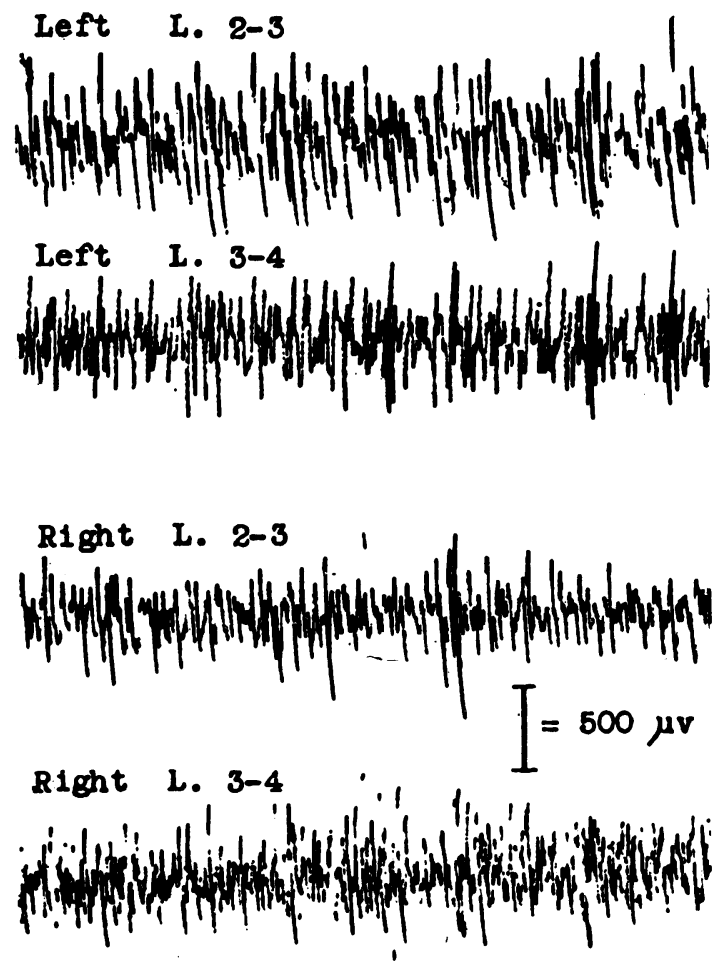

1 sec.

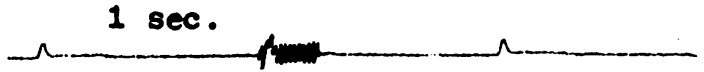

the lumbar spine. The patient is kept in the jacket until all pain has gone. When the jacket is removed he is instructed to perform extensor back exercises and taught how to lift avoiding flexion of the lumbar spine.

\section{Acknowledgments}

I would particularly like to thank Dr. P. H. S. Silver for his assistance with the electromyographic tracings, and Mr. W. F. Floyd for much technical advice. I am indebted to Mr. P. Wiles and Mr. P. H. Newman for their encouragement and permission to perform this work in their department.

\section{BIBLIOGRAPHY}

O'CONNELL, J. E. A. (1950), Ann. R.C.S., 6, 504.

MEYER, G. H. (1873) ' Die Statik und Mechanik des menschlichen Knochengerustes.' W. Engelmann, Leipzig.

AKERBLOM, B. V. (1948), 'Standing and Sitting Posture.' Nordiska, Stockholm.

FLOYD, W. F., and SILVER, P. H. S. (195 I), Lancet, i, I33.

NEWMAN, P. H. (1952), f. B. ft. Surg., 34 B, I, 30. 